\title{
Pulsations of microwave emission from a solar flare in a twisted loop caused by intrinsic magnetohydrodynamic oscillations
}

DOI:

10.1093/mnras/stac250

\section{Document Version}

Accepted author manuscript

Link to publication record in Manchester Research Explorer

Citation for published version (APA):

Smith, C., Gordovskyy, M., \& Browning, P. K. (2022). Pulsations of microwave emission from a solar flare in a twisted loop caused by intrinsic magnetohydrodynamic oscillations. Royal Astronomical Society. Monthly Notices, 511(2), 2880-2884. https://doi.org/10.1093/mnras/stac250

\section{Published in:}

Royal Astronomical Society. Monthly Notices

\section{Citing this paper}

Please note that where the full-text provided on Manchester Research Explorer is the Author Accepted Manuscript or Proof version this may differ from the final Published version. If citing, it is advised that you check and use the publisher's definitive version.

\section{General rights}

Copyright and moral rights for the publications made accessible in the Research Explorer are retained by the authors and/or other copyright owners and it is a condition of accessing publications that users recognise and abide by the legal requirements associated with these rights.

\section{Takedown policy}

If you believe that this document breaches copyright please refer to the University of Manchester's Takedown Procedures [http://man.ac.uk/04Y6Bo] or contact uml.scholarlycommunications@manchester.ac.uk providing relevant details, so we can investigate your claim.

\section{OPEN ACCESS}




\title{
Pulsations of microwave emission from a solar flare in a twisted loop caused by intrinsic MHD oscillations
}

\author{
Christopher Smith, ${ }^{1}$ M. Gordovskyy, ${ }^{1}$ and P.K. Browning ${ }^{1}$ \\ ${ }^{1}$ Department of Physics and Astronomy, University of Manchester, Oxford Road, Manchester M13 9PL, UK
}

In original form November 2021

\begin{abstract}
We present results revealing microwave pulsations produced in a model of a flaring twisted solar coronal loop, without any external oscillatory driver. Two types of oscillations are identified: slowly-decaying oscillations with a period of about $70-75 \mathrm{~s}$ and amplitude of about $5-10 \%$ seen in loops both with and without energetic electrons, and oscillations with period of about $40 \mathrm{~s}$ and amplitude of a few tens of percent observed only in loops with energetic electrons for about $100 \mathrm{~s}$ after onset of fast energy release. We interpret the longer-period oscillations as the result of a standing kink mode modulating the average magnetic field strength in the loop, whilst the short-period intermittent oscillations associated with energetic electrons are likely to be produced by fast variations of the electric field which produces energetic electrons in this scenario. The slowly-decaying oscillations can explain the quasi-periodic pulsations often observed in the flaring corona.
\end{abstract}

Key words: Sun: flares - Sun: oscillations - acceleration of particles

\section{INTRODUCTION}

Waves and oscillations are ubiquitous in the solar corona (e.g. De Moortel \& Nakariakov 2012; Nakariakov \& Kolotkov 2020). During flares and other active events, they can be observed in nearly all parts of the electromagnetic spectrum, from decametric radio to hard X-ray, with periods ranging from tens of milliseconds to tens of minutes (e.g. Nakariakov et al. 1999, 2004; Zimovets \& Struminsky 2009; Kumar et al. 2016). Understanding the origin and properties of these waves and oscillations is essential for understanding energy release and transport in solar flares. Furthermore, waves can serve as unique diagnostic tools for physical conditions in the solar corona (De Moortel \& Nakariakov 2012; Chen \& Peter 2015).

One type of oscillation observed in both solar and stellar flares is the so-called quasi-periodic pulsations (QPPs): intensity oscillations with typical periods in the range 1-100s and amplitudes 1-10\% observed in extreme UV, hard and soft X-ray, and microwave (MW) ranges. The exact nature of these oscillations is not fully understood and is a matter of heated debate (e.g. Van Doorsselaere et al. 2016; McLaughlin et al. 2018; Zimovets et al. 2021). Although the observed periods clearly indicate that QPPs are the result of some magnetohydrodynamic (MHD) process, it is not clear whether the observed oscillations are a direct effect of magnetic field oscillations in the corona or a proxy. For instance, the presence of QPPs in hard X-ray emission may indicate that MHD oscillations modulate particle acceleration in the corona, which, in turn, results in an oscillatory pattern in the intensity of bremsstrahlung produced by non-thermal electrons (e.g. McLaughlin et al. 2018; Carley et al. 2019; Hayes et al. 2019). Another key question is whether the observed QPPs are due to auto-oscillations of the coronal magnetic field structure, or instead forced or triggered externally, for example by an incoming MHD wave.

In this study, we address these questions through forward modelling, combining resistive-MHD and radiative transfer simulations, and using a semi-empirical model of the non-thermal electron population motivated by the results of MHD-test-particle simulations. We model a confined flare in a twisted coronal loop, in which energy is released by magnetic reconnection. We investigate the time-domain properties of the MW emission produced by this flaring loop, showing that pulsations similar in many ways to observed QPPs can be produced without any external wave-like modulation.

\section{THE MODEL}

The model of a confined solar flare in a twisted magnetic loop is based on the scenario developed by Gordovskyy \& Browning (2011); Gordovskyy et al. (2014) by combining 3D MHD and test-particle simulations (MHDTP Gordovskyy et al. 2010). In this model magnetic energy release is triggered by the onset of ideal kink instability (Browning et al. 2008). The instability leads to magnetic reconnection, heating the plasma (Hood et al. 2009) and accelerating electrons and ions (Gordovskyy et al. 

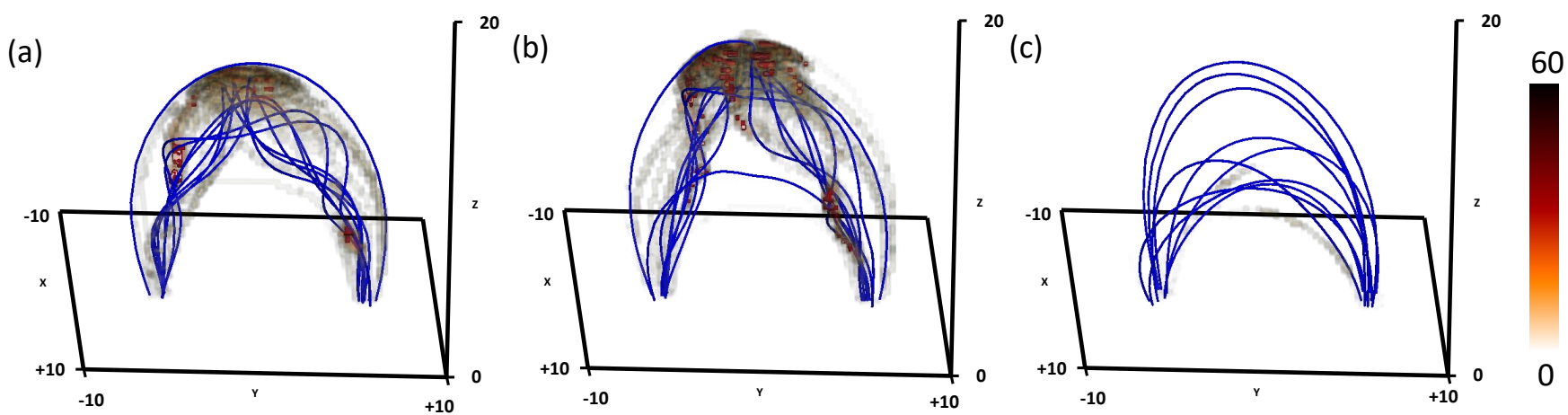

Figure 1. Evolution of the magnetic and electric field in the considered model. Blue lines denote selected magnetic field lines, while the semi-transparent volume plot show distribution of the parallel electric field in the domain. The corresponding colour scale shows values in $\mathrm{V} \mathrm{m}^{-1}$ units. Panels (a), (b) and (c) correspond to $\mathrm{t}=2 \mathrm{~s}, 80 \mathrm{~s}$ and $200 \mathrm{~s}$, respectively, after the onset of the kink instability.

2014). The properties of thermal and non-thermal emissions in this model have been studied by Gordovskyy et al. (2014, 2017) and Pinto et al. (2016).

We consider a twisted coronal loop with length of about $120 \mathrm{Mm}$, cross-section varying from about 4 Mm at foot-points to about $14 \mathrm{Mm}$ at the summit and the magnetic field varying from about $500 \mathrm{G}$ at the base to about $50 \mathrm{G}$ at the top. The loop is embedded within a gravitationally-stratified atmosphere with the temperature of $10^{4} \mathrm{~K}$ in a $2 \mathrm{Mm}$-wide layer just above the lower boundary of the domain (representing the chromosphere), and 0.9 MK everywhere else. The evolution of magnetic field and thermal plasma is calculated using 3D resistive-MHD LARE3D code (Arber et al. 2001) (Figure 1). At the onset of magnetic reconnection, the magnetic field is twisted with the twist angle of about $4 \pi$ (Figure 1a). Eventually, the loop kinks, resulting in localised enhancements of the current density, which, in turn triggers anomalous resistivity, leading to magnetic reconnection and energy release on a timescale of about $100 \mathrm{~s}$ (Figure 1b). The twist is reduced, lowering the magnetic energy, and the loop also becomes wider, due to reconnection between the loop field and the ambient field (Figure 1c). The high current densities and enhanced resistivity in some locations also result in high electric fields, which accelerates particles.

Gordovskyy et al. (2017) investigated the MW emission produced in this configuration and showed that the MW polarisation properties can be used as a diagnostic of the magnetic twist. This effect has subsequently been observed by Sharykin et al. (2018). In this study we use a similar approach, but focus on the time domain. We utilise a radiative transfer code built on the code by Fleishman \& Kuznetsov (2010). This code can simulate generation and transfer of free-free and GS emission from fast electrons in the MW spectral range, assuming that at every point within the model domain the energy distribution of electrons is as a sum of a Maxwellian distribution with density $n_{t}$ and temperature $T_{t}$, and a power-law non-thermal electron distribution with density $n_{b}$, power-law index $\gamma$, lower and upper energy cut-offs $E_{1}$ and $E_{2}$, respectively, analytical pitch-angle distribution.

The MW radiative transfer is modelled on a sparse numerical grid. The original $256 \times 256 \times 512$ grid from the MHD simulations is degraded to $64 \times 64 \times 64$ with the grid step of $1.25 \mathrm{Mm}$ in all directions. This substantially speeds-up calculations, while the resulting resolution $(\sim 1$ arcsec) is still better than most solar MW observations.

Using test-particles from MHDTP simulations directly would result in very high noise due to undersampling: it is impossible to evaluate the parameters of energetic electrons for each cell, given there may be only about 5 test-electrons per cell. Thus instead, energetic electron parameters are calculated analytically in a way which mimics the behaviour of energetic electrons in the MHDTP models (Gordovskyy et al. 2014; Pinto et al. 2016; Gordovskyy et al. 2017).

The spatial distribution of energetic electrons is determined by the amplitude and structure of the electric field component parallel to the magnetic field, which is calculated using Ohm's law: $E_{||}=\vec{B} \cdot(\eta \vec{j}-(\vec{v} \times \vec{B})) / B$. The number of energetic electrons in a given location $\vec{r}$ of the spatially-degraded grid is defined as $N(\vec{r})=0.01\left(\tilde{E}_{\|} / E_{t r}\right) \tilde{n_{t}}$, where $\tilde{n_{t}}$ and $\tilde{E}_{\|}$are the values of the thermal plasma density and parallel electric field averaged along the magnetic field. The above formula means that the fraction of electrons, that get accelerated is proportional to the local parallel electric field. The constant $E_{t r}$ is set to be $70 \mathrm{~V} \mathrm{~m}^{-1}$, this scaling factor is chosen to achieve a total fraction of non-thermal electrons typical of solar flares.

Averaging along the magnetic field reflects the fact that energetic electrons move very quickly, and, hence, redistribute along field lines on timescales by factor of about $v_{A} / c$ shorter than the timescale of processes in the MHD model (where $v_{A}$ and $c$ are the Alfven velocity and the speed of light, respectively). The process of deriving values of plasma density $\tilde{n_{t}}$, temperature $\tilde{T}_{t}$, and parallel electric field $\tilde{E}_{\|}$has been first implemented in Waterfall et al. (2020).

The energy and pitch-angle distribution of high-energy electrons is defined as $f \sim E^{-\gamma} \exp (-(\mu-1) / 0.04)$, limited by the lower and upper energy cut-offs, $E_{1}$ and $E_{2}$, respectively. Here $E$ is the electron kinetic energy, $\mu=v_{\|} / v$ is the pitch- 

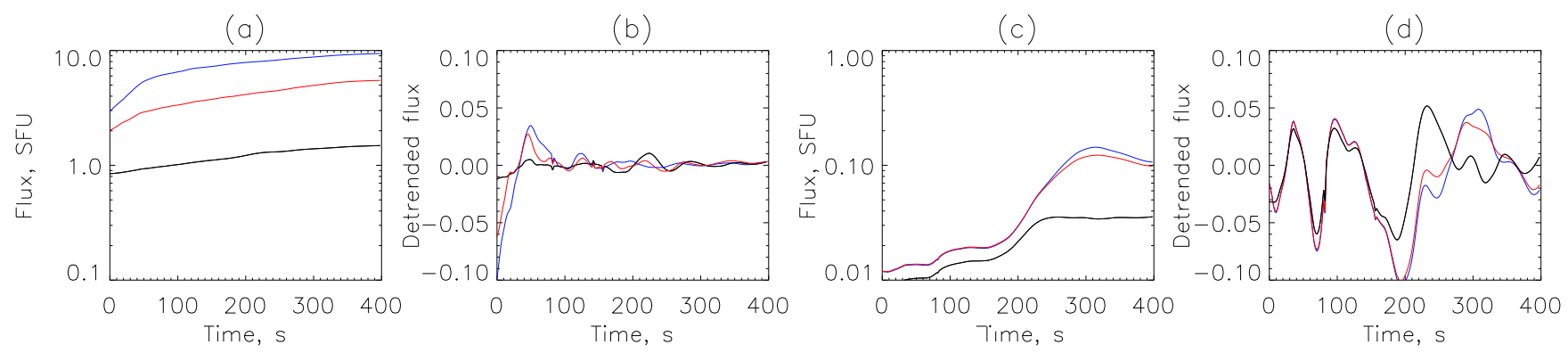

Figure 2. Light-curves (panels a and c) and de-trended light-curves (panels b and d) for the model with no particle acceleration. Horizontal axes show time after the onset of magnetic reconnection. Panels (a-b) and (c-d) correspond to the emission from the whole domains and loop-tops, respectively. Black, red and blue curves correspond to MW frequency of 5, 15 and 30 GHz. De-trended values (panels b and d) are normalised by the trend values.
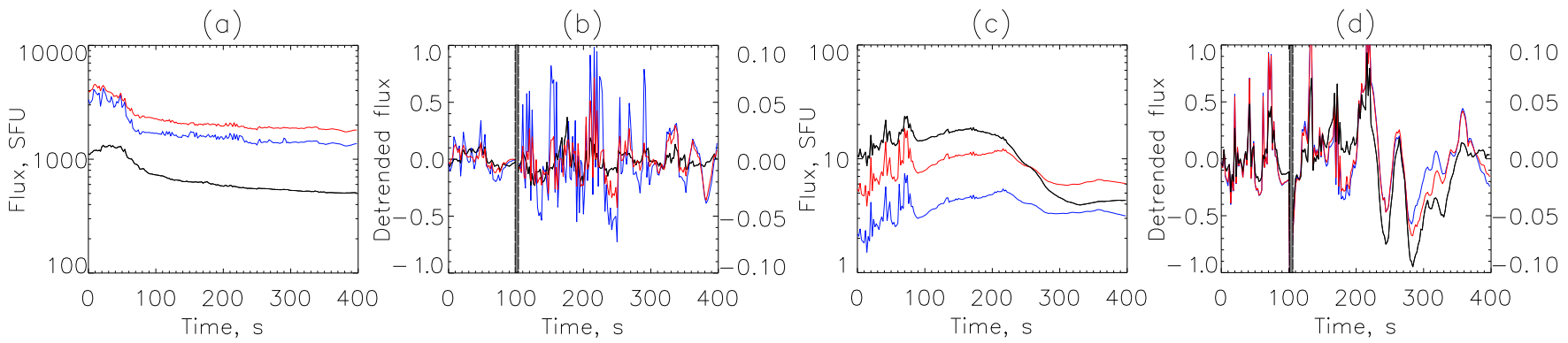

Figure 3. The same as in Figure 2, but for the model with non-thermal particles. There is a break in panels (b) and (d): de-trended values corresponding to times between $t=0$ and $t=100 \mathrm{~s}$ have different axis scaling.

(a)

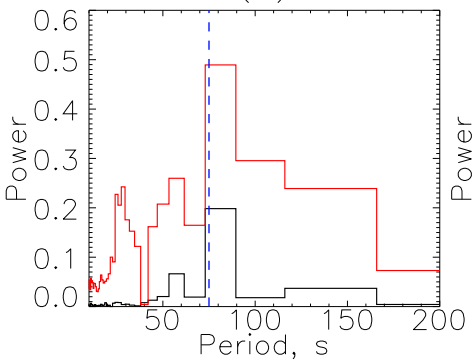

(b)

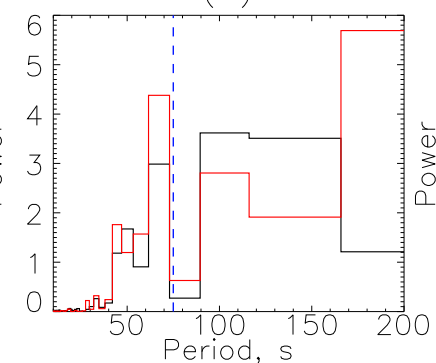

(c)

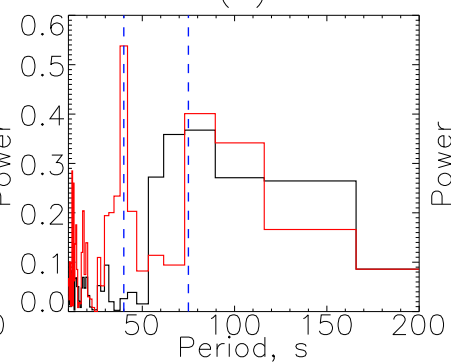

(d)

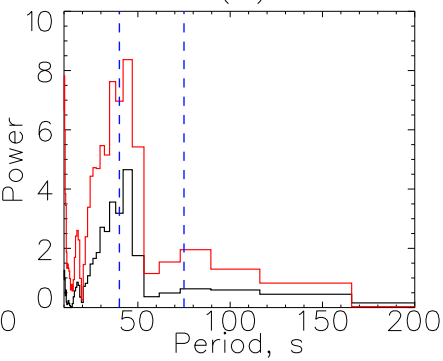

Figure 4. Power spectra of MW intensity oscillations. Panels (a-b) correspond to the fully thermal model, panels (c-d) correspond to the model with non-thermal particles as well as thermal plasma. Panels (a) and (c) correspond to the MW emission integrated over the whole domain, panels (b) and (d) correspond to the loop-top emission. The black and red lines are for MW frequency of 5 and 15 GHz, respectively. The blue dashed lines denote periods of 40 and $75 \mathrm{~s}$.

angle cosine, where $v_{\| \mid}$is the component of electron velocity parallel to magnetic field, $\gamma$ is power-law spectral index, $\Theta$ is the Heaviside (or step-like) function. The lower and upper energy cut-offs are taken to be $E_{1}=10 \mathrm{keV}$ and $E_{2}=10 \mathrm{MeV}$, respectively, at all times throughout the domain. The results in Section 3 are produced using electron energy distribution with $\gamma=3$.

The spatial structure of degraded parallel electric field $\tilde{E}_{\|}$is shown in Figure 1. Note the fragmented distribution of the parallel electric field, corresponding to the distribution of current sheets described above.

The observed radiation intensity (Stokes I) is forward-modelled assuming that the loop is viewed from the side, i.e. the MW intensity maps are produced for $y-z$ plane by integrating radiative transfer equations in $x$-direction. 


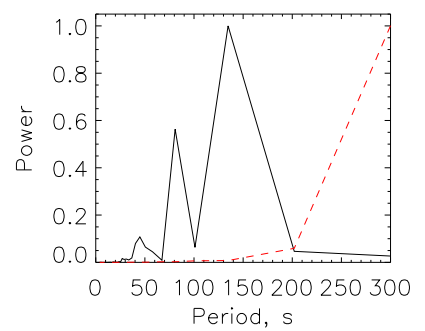

Figure 5. Power spectra of the LOS velocity variation (solid black line) and of average density variation (dashed red line) in the mid-plane of the loop.

\section{MICROWAVE INTENSITY OSCILLATIONS}

MW emission from the two models is calculated for frequency range 1-100GHz. In both models emission appears to be dominated by gyrosynchrotron. In the purely thermal model the spectral slope is positive in the whole considered spectral range. In the model with energetic particles, the spectral slop is positive under the turning frequency of about 30-35 GHz.

The study focuses on the lower frequencies, $5-30 \mathrm{GHz}$. This is because several instruments extensively used to study timedomain properties of MW emission in solar flares, such as Nobeyama Radio Heliograph, observed in this frequency range.

We analyse emission produced by the whole loop and, separately, by the loop-top region. The latter is bounded by heights of 32 and $48 \mathrm{Mm}$ above the lower boundary in the vertical $(z)$ direction, and by -8 and $+8 \mathrm{Mm}$ with respect to the loop summit location in the horizontal $(y)$ direction. MW light curves are shown in the left panels of Figure 2, for purely thermal plasma, and in Figure 3, for the case with non-thermal electrons in addition to the thermal plasma.

In order to detect any oscillatory signal, a de-trending procedure is carried out in a way similar to that used in observational data processing (e.g. Broomhall et al. 2019). The trend is determined by smoothing the original light-curves with the $100 \mathrm{~s}$ sliding window, with the edge values truncated. After the trend is subtracted, the remaining function is normalised by the trend function, giving the relative amplitude of the oscillatory component.

The characteristics of the MW emission from the purely thermal model are shown in the Figure 2. The MW intensities from this model slowly increase with time. This reflects the fact that the plasma heating, which is distributed within the loop due to plasma flows and thermal conduction, happens on relatively long timescales of about 200-300 s, in contrast with the Ohmic heating itself, which happens approximately within the first $100 \mathrm{~s}$ after onset of magnetic reconnection (Pinto et al. 2016). The increase of intensity from the loop-top is delayed compared to the intensity from the loop as a whole. This is because the energy release in this model is not concentrated near the loop top, it is distributed throughout the loop. In fact, because the magnetic field and current density are higher closer to the foot-points (because of the magnetic field convergence), most of the direct Ohmic heating occurs in the loop legs.

The de-trended lightcurves for the purely thermal case (Figure $2 \mathrm{~b}, \mathrm{~d}$ ) show distinct oscillations with a typical period in the range 50-100 s. Oscillations of the emission from the whole loop initially have an amplitude of about $5 \%$, but quickly decay, showing an amplitude of $1 \%$ after $300 \mathrm{~s}$ at all three considered frequencies $(5,15$ and $30 \mathrm{GHz})$. However, oscillations of the emission from at the loop-top show no signs of decay: the amplitude remains about $5 \%$ at all three frequencies. This difference between the loop-top and the whole loop is likely to be due to the fact that the oscillations of intensity produced in the loop legs is smeared by fast changes in the energy release and heating.

Oscillations of the emission coming from the loop top at the three different frequencies appear to be synchronised, unlike the intensity of the loop as a whole. This indicates that the radiation from the loop-top is mostly optically-thin, while the emission generated in the loop legs is optically-thick (see e.g. Gordovskyy et al. 2017). As the result, the loop-top emission 'samples' the same physical volume along the line-of-sight, while the emission from the loop legs at different frequencies 'samples' different layers of the loop.

The emission from the model containing non-thermal electrons is significantly different (Figure 3). Firstly, MW intensity peaks at about $100 \mathrm{~s}$ after the onset of the reconnection. After about $200 \mathrm{~s}$ it monotonically decreases. This reflects the fact that most of the emission is produced by energetic electrons, while particle acceleration is significant during the magnetic reconnection stage (about first $100 \mathrm{~s}$ ). During the first $\sim 100 \mathrm{~s}$, MW emission shows strong variations at all frequencies with the amplitudes of up to $50 \%$ with the characteristic period of $\sim 20-30 \mathrm{~s}$. The oscillations of MW intensity from the whole loop look almost like white noise, without any periodicity. At the same time, pulsations of MW emission from the loop-top seem to have a period of about 15-35 s. At the later stages, 100-400 s after onset of the reconnection, the loop lightcurves show oscillations with amplitude of about $5 \%$ and periods in the range 50-100 s, similar to the 'thermal' loop. However, in the model with non-thermal particles, these relatively low frequency oscillations are smeared with the higher frequency variations, yielding non-stationary pattern of pulsations, similar to those observed in some solar and stellar flares (Nakariakov et al. 2019). 
In order to understand time domain properties of the obtained lightcurves, we perform their Fourier analysis at each MW frequencies. The power spectra of the oscillations as functions of period are shown in Figure 4. As expected, the emission from the loop-top region in the purely thermal model reveals a relatively narrow, intense peak corresponding to period of around $60-70 \mathrm{~s}$. There is a significant signal at longer periods (>100-200 s), reflecting slower variations. In principle, these slow variations are the 'residuals' left by the de-trending procedure. It is impossible to clearly separate the trend and the oscillations because (a) there is no clear difference in periods and (b) the period of oscillations is in order-of-magnitude comparable to the modelling time interval, i.e. edge effects, inevitably, affect the power spectrum.

The power spectra corresponding to the whole domain also show a clear peak, although it is slightly shifted to longer periods, 70-80 s. Interestingly, both radio-frequencies shown in this graph, 5 and $15 \mathrm{GHz}$, have main peaks appearing at the same periods. This means that, although oscillations at different frequencies are not synchronised, this only affects the phase, but not the period.

The main difference between the power spectra corresponding to models with and without energetic particles is that the former model reveals additional peaks at shorter periods, about 40s. This peak corresponds to the oscillations, appearing during the first 100s. It is an interesting finding: although the corresponding interval of the light-curve seems to be quite noisy, the peaks are quite narrow.

The question regarding the nature of MHD oscillations resulting in the observed quasi-periodic MW pulsations remains open. The MW pulsations with the period of about 70-80 s are consistent with a standing MHD mode with the wavelength comparable to the loop length: the ratio of the loop length $(\sim 120 \mathrm{Mm})$ to the average Alfven speed $\left(\sim 1.4 \times 10^{6} \mathrm{~m} \mathrm{~s}^{-1}\right)$ is about $90 \mathrm{~s}$. The MW intensity can be modulated either by variation of the line-of-sight (LOS) component of the magnetic field due to the kink mode, or by variation of the number of energetic electrons due to sausage mode. The fact that we see clear pulsations in the nearly-optically-thin emission, at $30 \mathrm{GHz}$, from the loop-top in the case with energetic particles, indicates variation of the LOS component of magnetic field, meaning that MW pulsations are likely caused by the kink mode. Furthermore, analysis of the velocity field in the model reveals the presence of kink mode with a period close to the period of MW pulsations. Figure 5 compares power spectrum of the average LOS velocity in the mid-plane of the loop, which is a proxy for the kink mode, with the power spectrum of average density, which is a proxy of the sausage mode. The former shows peaks at 130-140 s and around $75 \mathrm{~s}$; both periods can result in the MW pulsations with the period of 70-75 s. At the same time, the density power spectrum has nearly power-law shape with no peaks. Hence, the longer period MW pulsations are most likely caused by the large-scale kink.

The nature of higher frequency oscillations in the model with energetic electrons is less clear. The sausage mode is more effective in modulating the current density and, hence, the parallel electric field, which, in turn, would modulate the number of energetic particles. The fact that its period is approximately half of the longer period oscillations may indicate that it is a higher harmonic global MHD mode modulating the average current density and parallel electric field.

\section{SUMMARY}

Our analysis of synthetic MW emission produced by thermal and non-thermal plasma in 3D magnetohydrodynamic simulations of reconnecting twisted coronal loops, finds MW intensity oscillations in the frequency range $5-30 \mathrm{GHz}$, without any external driver.

A simulated coronal loop with the length of about $120 \mathrm{Mm}$ and average magnetic field of about $70 \mathrm{G}$ exhibits oscillations with periods of about 70-80s and an amplitude of about 5\% during about 300-400s. Since these oscillations are observed in models both with and without energetic electrons, we interpret them to be due to a standing global MHD mode modulating the average magnetic field in the loop, which, in turn, modulates the GS emission produced by hot thermal plasma.

In addition to these oscillations, the loop with energetic electrons demonstrates an interval of higher-frequency oscillations with the period of about $40 \mathrm{~s}$. We interpret these oscillations as the result of a higher-order MHD mode modulating the average electric field in the loop, and, hence, modulating the number of energetic electrons, which produce GS emission during the energy release stage (about 100s).

Our key finding here is the existence of these oscillations, and this opens up the several significant areas for future work, including more detailed studies of observables. We believe that the kink mode is more likely to be responsible for the longer period MW pulsations, while fast oscillations observed immediately after onset of reconnection are caused either by higher-order kink or sausage mode. However, this issue will be addressed in more detail in a forthcoming study.

The obtained characteristics of the MW emission oscillations are very similar to those in solar flare quasi-periodic pulsations observed in the MW spectral range (e.g. Zimovets et al. 2021). Therefore, we conclude that auto-oscillations of a flaring loop can produce observed QPPs in the absence of any external oscillatory modulation. 


\section{ACKNOWLEDGMENT}

MG and PKB were supported by the Science and Technology Facilities Council (STFC, UK), grant ST/T00035X/1.

\section{DATA AVAILABILITY}

Lare3D code is available at warwick.ac.uk/fac/sci/physics/research/cfsa/people/tda/larexd/. The MW radiative transfer code is included into GX simulator framework, part of the SolarSoft, mssl.ucl.ac.uk/surf/sswdoc/solarsoft/ssw_install_howto.html. The data underlying this article will be shared on reasonable request to the corresponding author.

\section{REFERENCES}

Arber T. D., Longbottom A. W., Gerrard C. L., Milne A. M., 2001, Journal of Computational Physics, 171,151

Broomhall A.-M., et al., 2019, ApJS, 244, 44

Browning P. K., Gerrard C., Hood A. W., Kevis R., van der Linden R. A. M., 2008, A\&A, 485, 837

Carley E. P., Hayes L. A., Murray S. A., Morosan D. E., Shelley W., Vilmer N., Gallagher P. T., 2019, Nature Communications, 10,2276

Chen F., Peter H., 2015, A\&A, 581, A137

De Moortel I., Nakariakov V. M., 2012, Philosophical Transactions of the Royal Society of London Series A, 370,3193

Fleishman G. D., Kuznetsov A. A., 2010, ApJ, 721, 1127

Gordovskyy M., Browning P. K., 2011, ApJ, 729, 101

Gordovskyy M., Browning P. K., Vekstein G. E., 2010, ApJ, 720, 1603

Gordovskyy M., Browning P. K., Kontar E. P., Bian N. H., 2014, A\&A, 561, A72

Gordovskyy M., Browning P. K., Kontar E. P., 2017, A\&A, 604, A116

Hayes L. A., Gallagher P. T., Dennis B. R., Ireland J., Inglis A., Morosan D. E., 2019, ApJ, 875, 33

Hood A. W., Browning P. K., van der Linden R. A. M., 2009, A\&A, 506, 913

Kumar P., Nakariakov V. M., Cho K.-S., 2016, ApJ, 822, 7

McLaughlin J. A., Nakariakov V. M., Dominique M., Jelínek P., Takasao S., 2018, Space Sci. Rev., 214, 45

Nakariakov V. M., Kolotkov D. Y., 2020, ARA\&A, 58, 441

Nakariakov V. M., Ofman L., Deluca E. E., Roberts B., Davila J. M., 1999, Science, 285, 862

Nakariakov V. M., Arber T. D., Ault C. E., Katsiyannis A. C., Williams D. R., Keenan F. P., 2004, MNRAS, 349, 705

Nakariakov V. M., Kolotkov D. Y., Kupriyanova E. G., Mehta T., Pugh C. E., Lee D. H., Broomhall A. M., 2019, Plasma Physics and Controlled Fusion, 61, 014024

Pinto R. F., Gordovskyy M., Browning P. K., Vilmer N., 2016, A\&A, 585, A159

Sharykin I. N., Kuznetsov A. A., Myshyakov I. I., 2018, Sol. Phys., 293, 34

Van Doorsselaere T., Kupriyanova E. G., Yuan D., 2016, Sol. Phys., 291, 3143

Waterfall C. O. G., Browning P. K., Fuller G. A., Gordovskyy M., Orlando S., Reale F., 2020, MNRAS, 496, 2715

Zimovets I. V., Struminsky A. B., 2009, Sol. Phys., 258, 69

Zimovets I. V., et al., 2021, Space Sci. Rev., 217, 66 